\title{
Scanning Electron Microscopy With Slow Electrons.
}

\author{
Luděk Frank, Šárka Mikmeková, Zuzana Pokorná and Ilona Müllerová \\ Institute of Scientific Instruments AS CR, Brno, Czech Republic.
}

When focusing the primary electron beam in a scanning electron microscope (SEM) using a standard electromagnetic or electrostatic lens with the main aberration coefficients independent of the beam energy, we get a spot size proportional to (energy) $)^{-3 / 4}$ [1]. However, an immersion electrostatic lens has aberrations proportional to the lower of the electron energies existing on both its sides, so when retarding the primary beam with a bias applied directly to the sample via the cathode lens, we get a spot extending only as (energy) $)^{-1 / 4}$ at the lowest energies, with the overall spot size remaining within one order of magnitude throughout the full energy scale [1]. The above-sample field not only retards the primary electrons but also accelerates the signal electrons and collimates them towards the optical axis, thereby ensuring excellent collection efficiency for all energies. A below-sample detector enables performance of the transmission mode at any energy [2].

Scanning low energy electron microscopy (SLEEM) with a cathode lens provides improved image resolution at low energies, an enhanced signal of secondary electrons, a completely collected signal of backscattered electrons (BSE) including very low energy electrons that are traditionally abandoned, and plenty of contrast mechanisms not available with fast incident electrons. Below a fuzzy threshold at 50 $\mathrm{eV}$, the inelastic scattering phenomena fade away so the BSE signal strongly dominates and the penetration of primary electrons also increases. At and below hundreds of eV, the BSE signal abandons its dependence on the atomic density of the sample and becomes governed by its crystallinic structure, while at tens and units of $\mathrm{eV}$ the electronic structure is what reflects the image signal behavior.

From the experimental point of view, it is important to have flat samples with surface unevenness not exceeding tens of $\mu \mathrm{m}$ at hundreds of $\mathrm{eV}$ and units of $\mu \mathrm{m}$ at the lowest energies. It is good practice to cover the sample with a flat cap leveling the equipotential surface. With a primary beam energy of several keV we get, using the sample bias, excellent performance at lower energies, i.e. not only better resolution, but also much higher signal-to-noise ratio and enhanced surface sensitivity. When tuning the landing energy of electrons to what is known as the second critical energy, we get nonconductive samples imaged free of charging artifacts [3]. The dynamical theory of electron diffraction indicates [4], and practice confirms [5], that in the $10^{2} \mathrm{eV}$ range the grain contrast of polycrystals substantially increases and even the distribution of residual strains inside grains becomes visible [6]. Below $50 \mathrm{eV}$, the absorption of hot electrons injected into samples is low enough to allow the electron reflectance in the (00) spot to respond to the local density of empty electron states available in the direction of motion, which provides an alternative method of determining the local crystallographic system and its orientation [7]. After non-zero diffracted rays emerge, flat surface crystals may be imaged in the diffraction contrast [8]. When varying the landing energy of electrons, we get doped areas in semiconductors revealing either a proportional dopant contrast or a constant brightness [9], [10].

\section{References:}

[1] I Müllerová and L Frank, Adv. Imaging \& Electron Phys. 128 (2003), p. 309.

[2] I Müllerová et al, Ultramicroscopy 119 (2012), p. 78. 
[3] L Frank et al, Scanning 23 (2001), p. 36.

[4] L Reimer in "Scanning electron microscopy", (Springer, Berlin 1998) chapt. 9.

[5] Š Mikmeková et al, Materials Transactions 51 (2010), p. 292.

[6] L Frank et al, Materials 5 (2012), p. 2731.

[7] Z Pokorná et al, Applied Physics Letters 100 (2012), 261602.

[8] L Frank et al, Scanning 21 (1999), p. 1.

[9] M Hovorka et al, Materials Transactions 51 (2010), p. 237.

[10] The authors acknowledge funding from the Czech Science Foundation (grant no. P108/11/2270) and the Technology Agency of the Czech Republic (project no. TE01020118).
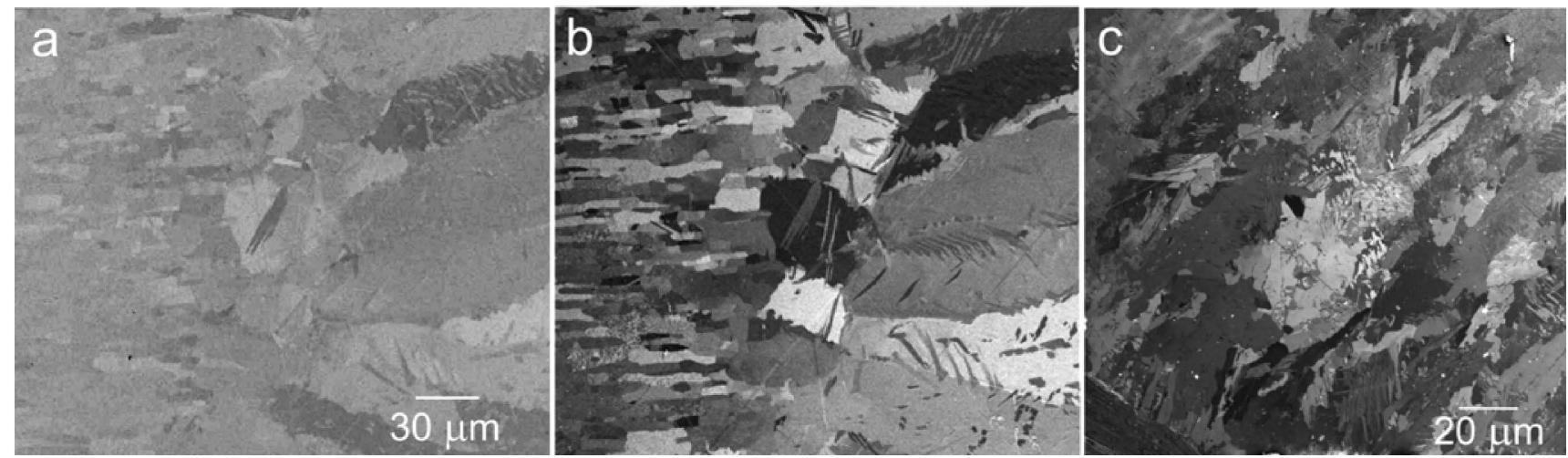

Figure 1. Microstructure of a laser beam weld in duplex (austenitic-ferritic) steel: margin of the seam imaged at $6 \mathrm{keV}$ in the BSE mode (a) and at $500 \mathrm{eV}$ in the SLEEM mode (b), seam centre at $500 \mathrm{eV}$ (c).
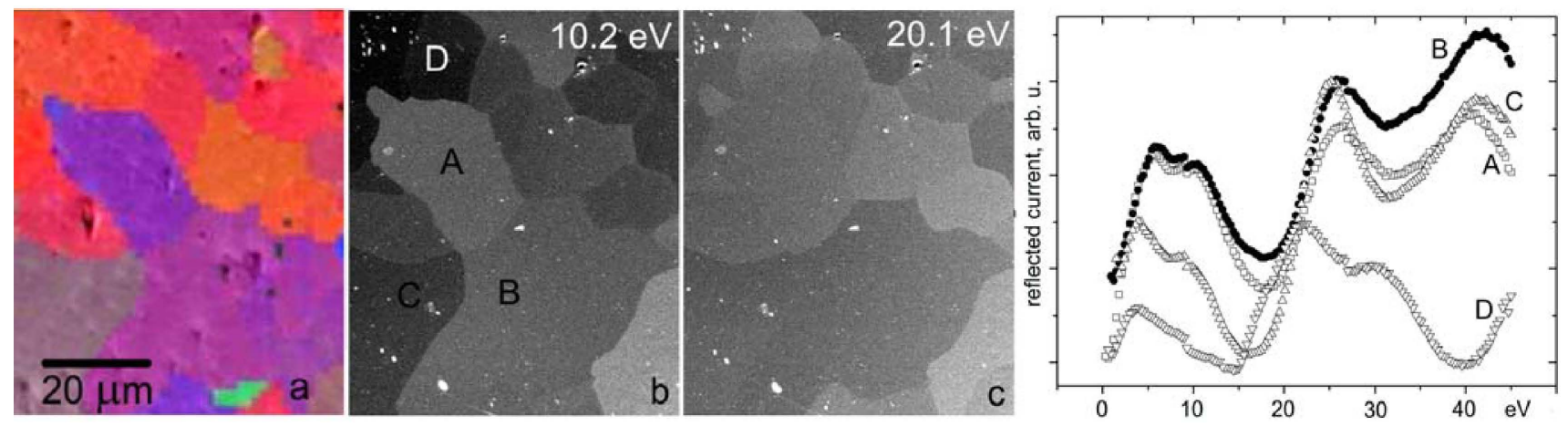

Figure 2. Identification of crystal grains in Al upon reflectance of very slow electrons: EBSD map (a), SLEEM frames (b), (c), energy dependence of the reflectance of selected grains (d).

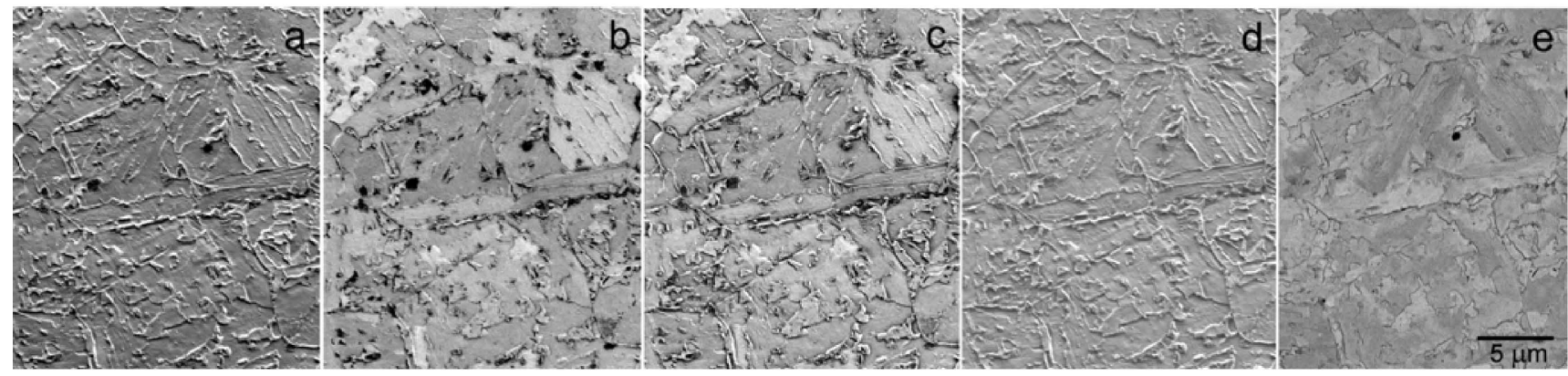

Figure 3. TRIP steel (bainite-ferrite with retained austenite), the SLEEM mode at $500 \mathrm{eV}$ (a) to (d), polar angles acquired: $17^{\circ}-30^{\circ}$ (a), $30^{\circ}-49^{\circ}$ (b), $49^{\circ}-72^{\circ}$ (c), $72^{\circ}-90^{\circ}$ (d); standard BSE mode, $4 \mathrm{keV}$ (e). 